PPPL- 2562

DE89 004623

\title{
Impurity Transport in Ohmically Heated TFTR Plasmas
}

\author{
B. C. Stratton, R. J. Fonck, R. A. Hulse, A. T. Ramsey, J. Timberlake, \\ P. C. Efthimion, E. Fredrickson, B. Grek, K. W. Hill, D. W' 'ohnson, \\ D. K. Mansfield, H. Park, F. J. Stauffer*, and G. Tayı \\ Princeton Plasma Physics Laboratory, Princeton University, Princeton, NJ, 08543
}

\begin{abstract}
Impurity transpon in ohmically heated TFTR plasmas is studied by cc nputer modeling of VUV line emissions from impurities injected using the laser- lowoff technique. The results are sensitive to uncertainties in the ionization and recombination rates used in the modeling; as a result, only a spatially averaged diffusion coefficient and parameterized convective velocity can be measured. Measurements of these transport parameters are presented for deuterium and helium discharges with $\mathrm{I}_{\mathrm{p}}=0.8-2.5 \mathrm{MA}, \overline{\mathrm{n}_{\mathrm{e}}}=0.6-6.0 \times 10^{19} \mathrm{~m}^{-3}$, and $\mathrm{Z}_{\mathrm{eff}}=2-6$. Diffusion coefficients are found to be in the $0.5-1.5 \mathrm{~m}^{2} / \mathrm{s}$ range, considerably larger than neoclassical values. Nonzero inward convective velocities are necessary to fit the data in most cases. No dependence of the diffusion coefficient on injected element, working gas species, or plasma current is found, but at a given current, the diffusion coefficient is smaller by approximately a factor of two in plasmas near the density limit than in discharges with $\overline{n_{e}}<3 \times 10^{19} \mathrm{~m}^{-3}$.
\end{abstract}

*Present address: AT\&T Microelectronics, Reading, PA, 19612

\section{MASTER}




\section{Introduction}

Particle transport plays an important role in determining the confinement properties of tokamak plasmas and has therefore been studied on a number of tokamaks. In particular, measurements of impurity transport in a number of smalland medium-sized tokamaks have yielded diffusion coefficients on the order of 1 $\mathrm{m}^{2} / \mathrm{s}$ and, in many cases, significant inward convective velocities. The measured diffusion coefficients were often found to be significantly larger than the neoclassical values.

Only a few measurements of particle transport have been made in the current generation of large tokamaks. In TFTR, transport parameters of the same magnitude as those described above were found for injected germanium [1], a helium gas puff [2], and a deuterium puff [3]. In JET [4] and JT-60 [5], similar values of the diffusion coefficient were deduced from iron and titanium injections. These measurements cover only a limited range of discharge conditions. Because understanding particle transport is important to tokamak physics, it is valuable to measure impurity transport parameters over a wide range of discharge conditions and to look for trends.

This paper presents impurity transport measurements in ohmically heated TFTR discharges which covered a wide range of values of plasma current $\left(I_{p}\right)$, electron density $\left(n_{e}\right)$, and used both deuterium and helium as the working gas. The transpor parameters were deduced by MIST [6] impurity transport code modeling of VUV line emissions from impurities injected using the laser-blowoff technique [7]. The results are compared with a neoclassical impurity transport calculation. In addition to providing documentation of impurity transport in TFTR discharges with ohmic heating only, this paper provides comparison data for furure work on impurity transport in TFTR plasmas with beam heating and pellet fuelling.

Section 2 of this paper describes the observed plasmas, the impurity injection technique, and the spectroscopic instrumentation used. Section 3 describes the MIST code modeling of the data. Section 4 gives a detailed example of the data and analysis, and discusses a problematic aspect of the modeling: lines from ionization states near the plasma edge are observed to have longer decay times than predicted by a transport model that fits the central ionization state data well. The sensitivity of the deduced transport parameters to uncertainties in the ionization and recombination rates used in the modeling, the probable cause of this problem, are 
discussed. In section $\mathbf{5}$, the data from the example discharge are compared with a neoclassical transport calculation, and the diffusion coetficient and convective velocity from the example discharge are compared with a measurement of the electron diffusion coefficient and convective velocity. Section 6 presents measurements of transport parameters for 17 ohmically heated deuterium and helium discharges with $\mathrm{I}_{\mathrm{p}}$ in the range $0.8-2.5 \mathrm{MA}$ and $\overline{\mathrm{n}_{\mathrm{e}}}=0.6-6.0 \times 10^{19} \mathrm{~m}^{-3}$, and the results are summarized in section 7 .

\section{TFTR parameters and diagnostics}

The plasmas studied here were standard full-size TFTR discharges with $\mathrm{R}=2.36-2.59 \mathrm{~m}$ and $\mathrm{a}=0.71-0.83 \mathrm{~m}$ (such plasmas are described in detail in ref. 8 ). Two of the discharges were limited by a moveable poloidal limiter on the large major radius side of the plasma; the remainder were limited by a toroidal inner-wall limiter. Both limiters were graphite. $\mathrm{Z}_{\text {eff }}$ measured from visible bremsstrahlung emission was 2.0-6.0. and. except in the highest density plasmas, carbon was the dominant impurity species. In the highest density plasmas carbon and oxygen contributed approximately equally to $Z_{\text {eff }}$. The toroidal field $\left(B_{\mathfrak{t}}\right)$ in these discharges was 3.9-4.9 T. (Plasma parameters for each shot are listed in Table III below.)

Impurities were injected using the taser-blowoff technique [7]: a thin metallic film on a glass slide is illuminated from behind by a ruby laser pulse, and material is ablated: both individual atoms and clusters of atoms are produced and enter the plasma. The injections were done well into the plasma current flat top when the plasma was near equilibrium. $\mathrm{T}_{\mathrm{e}}(0)$ is $1.5-5 \mathrm{keV}$ in TFTR plasmas with ohmic heating only, and MIST calculations for these plasmas show that the distributions of the $\mathrm{Li}$ - and $\mathrm{Be}$-like ionization states are peaked on axis in elements with $\mathrm{Z} \sim 30$; lines emitted by these states are easily observed in the 50-300 $\AA$ region. Thus, germanium $(Z=32)$ was used for most of the present work, with other elements being used in several shots.

Lines emitted by the Mg-, Na-, Be-, and Li- like ions of the injected element were observed using the SPRED [9] and SOXMOS [10] spectrometers. Using a $450 \mathrm{~g} / \mathrm{mm}$ grating, SPRED observes the $100-1100 \AA$ region with $2 \AA$ resolution. The line brightnesses were measured with SPRED, which has been radiometrically calibrated [11] using synchrotron radiation from the National Bureau of Standards 
SURF II facility, and using branching ratios. SOXMOS covers the 10-320 $\AA$ region with $\sim 0.2 \AA$ resolution; $25-60 \AA$ of this range can be observed in a single discharge. SPRED detector integration times were $5-20 \mathrm{~ms}$, and SOXMOS integration times were $25-50 \mathrm{~ms}$.

\section{Impurity Transport Modeling}

The data are modeled using MIST [6], a one-dimensional radial impurity transport code which solves the impurity continuity equations

$$
\frac{\partial}{\partial t} n_{\mathrm{q}}=-\frac{1}{r} \frac{\partial}{\partial r}\left(r \Gamma_{q}\right)+I_{q-1} n_{q-1}-\left(I_{q}+R_{q}\right) n_{q}+R_{q+1} n_{q+1}-n_{q} / \tau_{q}+S_{q}
$$

where $\mathrm{n}_{\mathrm{q}}$ is the impurity density in ionization state $\mathrm{q}, \Gamma_{\mathrm{q}}$ is the impurity flux density, and $I_{q}$ and $R_{q}$ are ionization and recombination rates, respectively. $S_{q}$ describes the impurity source, which is assumed in this work to be an instantaneous burst of monoenergetic impurity atoms at $3 \mathrm{eV}$. Particle losses are described by $\tau_{\mathrm{q}}$. a confinement time for parallel flow to the limiter in the scrape-off region. $\tau_{q}$ is parameterized as $\lambda^{2} / \mathrm{D}$, where $\lambda$ is a scale length describing the effective width of the scrape-off region, and $D$ is the impurity diffusion coefficient. While $\lambda=0.1 \mathrm{~m}$ was used in the present work, variations of $\lambda$ in the range $0-0.1 \mathrm{~m}$ show that the results are insensitive to the specific value chosen.

The electron impact ionization, dielectronic recombination, and radiative recombination rates used in MIST are calculated as described by Post et al. [12]. The rates are evaluated using measured $T_{e}$ and $n_{e}$ profiles. Charge-exchange recombination [13] is also included; thermal neutral densities and temperatures are calculated by SNAP, a kinetic thermal transport code [14]. The line brightnesses are calculated from the Van Regemorter formula for electron impact excitation and Mewe's gaunt factors [15].

$\Gamma_{\mathrm{q}}$ is assumed to be the sum of diffusive and convective terms:

$$
\Gamma_{\mathrm{q}}=-\mathrm{D}_{\mathrm{q}}(\mathrm{r}) \frac{\partial}{\partial \mathrm{r}} \mathrm{n}_{\mathrm{q}}(\mathrm{r})+\mathrm{v}_{\mathrm{q}}(\mathrm{r}) \mathrm{n}_{\mathrm{q}}(\mathrm{r})
$$

where $D_{q}$ is a diffusion coefficient and $v_{q}$ is a convective velocity. Note that neoclassical fluxes may also be written in this general form. In principle, the functional forms of $D_{q}$ and $v_{q}$ may be deduced from modeling of the time 
evolutions of line brighenesses from a number of ions existing at different locations in the plasma. In practice, the data quality and uncertainties in the modeling do not permit this to be done uniquely. Assumptions for the functional forms of $D_{q}$ and $v_{q}$ are therefore necessary. $D$ and $v$ are assumed to be independent of impurity ionization state. and $\mathrm{D}$ is assumed to be constant as a function of minor radius. The convective velocity is parameterized as

$$
v(r)=c_{v} D(r) \frac{\partial}{\partial t} \ln \left(n_{e}(r)\right)
$$

where $c_{v}$ is a peaking parameter which is adjusted to fit the data. The significance of this parameterization may be seen from an equilibrium solution to $\mathrm{Eq}$. (1). In equilibrium, the shape of the total impurity density profile in the source/sink free region, $n_{z}(r)=\sum n_{q}(r)$, is simply related to $v(r) / D(r)[16]$. Because $D$ is assumed to be constant, the shape of $n_{z}(r)$ is determined by $v(r)$. If $v(r)$ is parameterized according to Eq. (3), $n_{Z}(r)$ has the shape of $n_{e}(r)$ to the $c_{v}$ power. This parameterization is useful because it allows the equilibrium impurity density profile to be compared to the $\mathrm{n}_{\mathrm{e}}$ profile in a simple way.

\section{Example of Data Modeling and Sensitivity to Atomic Rates}

As an example of the data and modeling, results from a set of data obtained in 1.4 MA deuterium discharges at $\overline{n_{e}}=1.2 \times 10^{19} \mathrm{~m}^{-3}$ will be discussed in detail. Figure 1 shows the time evolutions of $\mathrm{I}_{\mathrm{p}}, \overline{\mathrm{n}_{\mathrm{e}}}$, the loop voltage, and the total radiated power in one of these discharges. A small amount of germanium was injected at $4.1 \mathrm{sec}$. well into the $I_{p}$ flat top; note that the changes in the loop voltage and total radiated power caused by the injection are small. $T_{e}(0)$ at this time was $4.2 \mathrm{keV}$. These discharges also had a small deuterium puff starting at $3.0 \mathrm{~s}$ and lasting for $0.05 \mathrm{~s}$. whose purpose was to permit a measurement of the electron diffusion coefficient and convective velocity to be made; the results of this measurement are compared with the impurity transport parameters below.

The time evolutions and brightnesses of the Ge XXI $196 \AA$, Ge XXII $226 \AA$, Ge XXX $123 \AA$, and Ge XXX $200 \AA$ lines were measured in these discharges. To simplify discussion, only one line from an edge ionization state, Ge XXI $226 \AA$, and one from a central state, Ge XXX $260 \AA$, are discussed in detail; comparison of the data and calculations is similar for the other two lines. Figure 2 shows the measured and calculated time evolutions of the Ge XXII $226 \AA$ and Ge XXX $200 \AA$ lines: data 
from three identical shots were averaged together. (The Ge XXX line shows an enrly peak due to emission from low ionization states of germanium which should be ignored.) The calculations assumed $D=i .3 \mathrm{~m}^{2} / \mathrm{s}, \mathrm{c}_{\mathrm{v}}=0.3$. and $1.0 \times 10^{17}$ injected atoms and were normalized to the measurements at the time of peak brightness for each line. It is clear that, while the fit to the Ge XXX data is good. the measured decay time of the Ge XXII line is significantly longer than the calculated value. as is also true for the Ge XXI line. Figure 3 shows the calculated distribution of ionization states for this transport model $0.2 \mathrm{~s}$ after injection: the $\mathrm{Ge}^{+29}$ distribution is peaked on axis and the $\mathrm{Ge}^{+20}$ and $\mathrm{Ge}^{+2 \mathrm{l}}$ distributions peak in $\mathrm{t}^{2}$ : 0.6-0.7 $\mathrm{m}$ region, near the plasma edra. (For clarity. states below $\mathrm{Ge}^{+20}$ are not shown.) Table I lists the measured and calculated line brightnesses $0.1 \mathrm{~s}$ after injection. The agreement between measurement and calculation is indicated by the ratio of measured to calculated brightnesses: on average, brightnesses of lines emitted by the edge states are somewhat larger than predicted.

For a given parameterization of the transpon parameters. variations of the diffusion coefficient and peaking parameter affect differently the calculated rise and decay times of lines emitted by central ionization states such as Ge XXX: increasing the diffusion coefficient decreases both the rise and decay times. while increasing the peaking parameter causes a small increase in the rise time and a much larger increase in the decay time. The reason for this is that the diffusive term dominates the flux at early times due to the large impurity density gradient. The effects of diffusion and convection can therefore be separated to some extent in modeling the central ionization states. In contrast, the rise times of the edge states are almost entirely determined by ionization since little time is required for the particles to move a few centimeters into the plasma, and their decay times are determined by ionization, recombination, and transport. The transport model of Fig. 2 was chosen to reproduce the rise and decay times of the Ge XXX line, and as previously mentioned, the observed decay times of the Ge XXII and Ge XXI lines are significantly longer than the calculated ones. Different values of the diffusion coefficient and peaking parameter, as well as diffusion coefficients with various radial dependences, have been tried, but it does not appear to be possible to find a transport model which reproduces the time evolutions of lines from both the central and edge ionization states well. This is a general problem in modeling TFTR impurity injection data. Long decay times for edge ionization states have also been seen in impurity injection experiments on smaller tokamaks [17]. 
The probable caluse of the discrepancv between the calculated and measured time evolutions of lines from the edge ionization states is inaccurate ionization and recombination rates. As an example of the sensitivity of the modeling to variations in these rates. Figs. $t$ and 5 show the measured time evolutions of the germanium lines in the example discharge along with calculations using the same transport model as in Fig. 2, with the ionization and recombination rates multiplied and divided by two. It is clear that varying the rates has a significant effect on the calculated time evolutions of the lines, particularly for the edge ionization states. Varying the ionization rates changes the rise time by almost $100 \%$ for thie edge states and by $30.40 \%$ for the central states. The effect on the rise times of varying the recombination rates is less pronounced: the rise times of the edge states change by less than $25 \%$ and those of the central states vary by less than $15 \%$. The effect of varying the rates on the decay times is quite different. Decreasing the ionization rates or increasing the recombination rates increases the decay times of the edge states by a factor of 2 , while increasing the ionization rates or decreasing the recombination rates decreases the decay times of the edge states by less than $35 \%$. The decay times of the central states are virtually unaffected by variations in the rates because, at times later than the peak of the emission from the central states. transport is slower than ionization and recombination of these states.

The rate variations also cause changes in the calculated brightnesses. Table II shows the ratio of the calculated line brightness to the teference values (the calculated values in Table I) for the same atomic rate variations. (Variations in the brightness of the Ge XXX $123 \AA$ line, not shown, follow those of the Ge XXX $200 \AA$ line.) Increasing the ionization rates or decreasing the recombination rates decreases the brightnesses of the edge lines by a factor of 3 to 5 , while the brightnesses of the central lines are unaffected. Decreasing the ionization rates or raising the recombination rates increases the brightnesses of the edge lines by a factor of 1 to 3 , and reduces the brightnesses of the central lines by a factor of 2 . Thus, although agreement between the measured and calculated brighmesses in Table I is reasonable, the rate variations show that the brightnesses do not provide a useful constraint on the transport model.

Figures 4 and 5 show that the measured and calculated decay times of the edge states can be brought into good agreement by decreasing the ionization rates or by increasing the recombination rates by a factor of two. For the ionization rate decrease, a small decrease in the diffusion coefficient is necessary to make the 
calculated and measured rise times of the central states agree: little variation in the transport is needed in the case of the recombination rate increase. Similar variations of the rates for other TFTR impurity injection data yield transport models which fit both the edge and central states well.

Few measurements or ab initio salculations of electron-impact ionization and dielectronic recombination rates exist for elements with Z $>28$ : thus, MIST uses semi-empirical formulae similar to those of Lotz [18] and Merts [19] for the electron-impact jonization and dielectronic recombination rates. respectively. The accuracy of the Lotz and Burgess-Merts formulae is discussed by Janev and Katsonis [20]. They were derived trom data for elements with lower $Z$ than considered here. and are not considered to be accurate for elements with Z 30. In particular, the Lotz formula significantly overestimates the direct ionization rate for $Z>20$. However, excitation followed by autoionization can be an important process for ions up to Na-like. and the Lotz formula underestimates the measured total ionization rate for these ions by approximately a factor of two. For higher jonization states. the Lotz formula is approximately correct [21]. Note that this indicates that the ionization rates should be increased, which would produce even poorer agreement with the edge ionization state data. Comparison of the Burgess-Merts formula with the few existing measurements and ab initio calculations of dielectronic recombination rates shows that this formula generaliy underestimates the rates, sometimes by more than a factor of two [20]. Increasing these rates produces better agreement with the low ionization state data. Factor-of-two variations in the atomic tates are therefore reasonable, and, in parti-ular, it is reasonable to increase the recombination rates by a factor of two.

Another possible, although unlikely, explanation of the long decays of the lines from low ionization states is a prolonged impurity source; such a source has been used in modeling TFR impurity injection data [17]. MIST modeling indicates that a source which decays in time following the initial injection and persists for several hundred milliseconds reproduces the time evolutions of the edge ionization state lines in TFTR. However, there is no evidence of recycling or deposition followed by erosion of injected impurities in TFTR [22]: lines of injected impurities are not seen prior to injection in the succeeding discharge, and calculations show that the fraction of metallic impurity ions backscattered by a graphite target is small [23]. Injected impurities leaving the plasma are likely to be buried too deep in the limiter graphite to be released during the same discharge, and the sputtering rate for those 
deposited on the limiter surface is low [24]. Therefore, a prolonged impurity source is not a likely explanation of the long decays of the edge states.

Changes in the ionization and recombination rates due to the perturbation of $\mathrm{T}_{\mathrm{e}}$ caused by the injection itself has been examined as a possible cause of the long decay times of the edge ionization states. In general. the amount of medium- $Z$ impurity injection required to produce useable spectroscopic data caused perturbations in global plasma parameters such as $\mathrm{T}_{\mathrm{e}}$ and the radiated power. An extreme example is a 1.4 MA helium discharge at $\overline{n_{e}}=2.1 \times 10^{19} \mathrm{~m}^{-3}$ in which the injection increased the total radiated power from $0.8 \mathrm{MW}$ to $1.5 \mathrm{MW}$. Electron-cyclotron-emission measurements show that $\mathrm{T}_{\mathrm{e}}(0)$ dropped from $3.33 \mathrm{keV}$ before injection to a minimum of $3.15 \mathrm{keV} 0.1 \mathrm{~s}$ after injection and recovered to the pre-injection value $0.4 \mathrm{~s}$ after injection. At $\mathrm{r}=0.6 \mathrm{~m}$, the largest minor radius at which the measurements were reliable, $T_{e}$ dropped from $0.79 \mathrm{keV}$ before injection to a minimum of $0.68 \mathrm{keV} 0.02 \mathrm{~s}$ after injection and recovered in $0.4 \mathrm{~s}$. The perturbation of the $n_{e}$ profile was negligible. MIST calculations using time-dependent $T_{e}$ and $n_{e}$ profiles showed less than $10 \%$ difference in the rise and decay times of the lines compared to calculations using the same transport model and the $T_{e}$ and $n_{e}$ profiles before injection. Thus. changes in the atomic rates due to the $T_{e}$ drop caused by the injection cannot explain the persistence of the edge ionization states.

It is also conceivable that the perturbation of the plasma by the injection could cause a change in the transport, i.e., the transport parameters depend on the amount of injected impurity. Comparison of data from discharges in which the amount injected differed by a factor of two showed no difference in the time evolutions of the lines. However, even the smaller injected amounts caused some perturbation of the plasma and thus could still be affecting the transport.

In summary, inaccuracies in the ionization and recombination rates used in the code appear to be the most satisfactory explanation of the discrepancy between the measured and calculated decays of the edge ionization states. The high sensitivity of edge state lines to the rates implies that only the central state lines provide useable transport information. Thus, in the present work the diffusion coefficient and peaking parameter were obtained by fitting only data of the central ionization states. Such transport models yield a global value of the diffusion coefficient, i. e.. diffusion coefficients with different radial dependencies but the same average value cannot be distinguished. Rate variations and data quality in the example discharge 
and in the other discharges studied here indicate that the estimated accuracy of the diffusion coefficient derived from a two-parameter core transport model (D, $c_{v}$ ) is $-30-40 \%$. As seen from the range of values listed in Table III below. the convective peaking parameter is less accurately determined.

\section{Comparison with Neoclassical Transport Calculations and Electron Transport Coefficients}

Neoclassical transport alone is not adequate to describe the data. although the neoclassical convectivc velocities are similar to those derived from best fits to the data. This is illustrated by Fig. 6 which shows a comparison of the example data with a neoclassical transport calculation [25] in which both the impurity ions and the deuterons are assumed to be in the Pfirsch-Schlüter regime. This assumption is incorrect for the deuterons, which are actually in the banana regime, but should not change the results significantly since collisions between the injected impurities and background carbon ions, which are included in the calculation, dominate the transport for these calculations. The carbon concentration was taken from the measured $Z_{\text {eff }}$, and a flat profile was assumed. The ion temperature profile was calculated using the SNAP code [14] assuming neoclassical ion thermal conductivity. It is clear from Fig. 6 that neoclassical transport alone does not describe the data: the small neoclassical diffusion coefficient, $\sim 0.1 \mathrm{~m}^{2} / \mathrm{s}$ at the plasma edge and decreasing to $0.004 \mathrm{~m}^{2} / \mathrm{s}$ toward the center, yields rise and decay times that are much too long (the Ge XXX line intensity peaks at $2 \mathrm{~s}$ ). This discrepancy has been observed in other TFTR discharges. As seen in Fig. 6, neoclassical transport plus an "anomalous" diffusion coefficient of $1.1 \mathrm{~m}^{2} / \mathrm{s}$, close to the value used in Fig. 2. reproduces the Ge XXX data well. The neoclassical convective velocity for $\mathrm{Ge}^{+29}$ and that obtained from $c_{\mathrm{V}}=0.3$ have similar values of $\sim 10 \mathrm{~m} / \mathrm{s}$ near the edge and drop to less than $1 \mathrm{~m} / \mathrm{s}$ inside $r=0.65 \mathrm{~m}$. Thus, it is possible that neoclassical convection is adequate to provide the observed convective velocity.

The transport coefficients of the electrons were similar to the je of the impurities in the example discharge. The time evolution of the electron density profile following a deuterium puff similar to that shown in Fig. 1 was modeled to obtain a measurement of the electron diffusion coefficient and convective velocity in deuterium discharges with $\mathrm{I}_{\mathrm{p}}=1.4 \mathrm{MA}$ and $\overline{\mathrm{n}_{\mathrm{e}}}=1.2 \times 10^{19} \mathrm{~m}^{-3}$ [3]. These measurements yielded a hollow diffusion coefficient with values of $0.4 \mathrm{~m}^{2} / \mathrm{s}$ in the 
plasma core and $1.0 \mathrm{~m}^{2} / \mathrm{s}$ at $\mathrm{r} / \mathrm{a}=0.75$. and a convective velocity of $0.1 \mathrm{~m} / \mathrm{s}$ in the core and $4 \mathrm{~m} / \mathrm{s}$ at $\mathrm{r} / \mathrm{a}=0.75$. Thus, the electron and impurity transport coefficients are roughly similar in this case.

\section{Impurity Transport in Different Discharge Conditions}

The diffusion coefficient does not depend strongly on global discharge conditions. Table III lists the transport parameters and plasma conditions for 17 discharges with impurity injection. Tr. injected element, working gas, $I_{p}, B_{t}, \overline{n_{e}}$, and $Z_{e f f}$ at the time of injection are given. $D$ and $c_{v}$ were ubtained by modeling line intensities of the central ionization states as described above and, in some cases, a range of values which gave acceptable fits to the data is listed to serve as an indicator of the accuracy of the results. For consistency in these cases, the larger value of $D$, which corresponds to the larger value of $c_{v}$, is used for comparison with values from other discharges in the discussion below. Also listed is $\tau$, the global impurity decay time measured from the time evolution of a line emitted by a central ionization state or from the central-chord soft $x$-ray data.

Table III shows that diffusion coefficients in the range $0.5-1.5 \mathrm{~m}^{2} / \mathrm{s}$ are found for deuterium and helium plasmas with $\mathrm{I}_{\mathrm{p}}=0.8-2.5 \mathrm{MA}$ and $\overline{\mathrm{n}_{\mathrm{e}}}=0.6-6.0 \times 10^{19} \mathrm{~m}^{-3}$. Thus, the diffusion coefficient does not depend strongly on global discharge conditions. Nonzero convection is required in most cases (the nonzero values of $c_{V}$ given in Table III correspond to $v=2-10 \mathrm{~m} / \mathrm{s}$ at the limiter radius).

Comparing injections of $\mathrm{Ti}, \mathrm{Ge}$, and $\mathrm{Mo}$ into 1.4 MA helium discharges at $\overline{\mathrm{n}_{\mathrm{e}}}=1.8-2.2 \times 10^{19} \mathrm{~m}^{-3}$ (cases 3, 12 , and 13 in Table III) shows no strong dependence of $D$ on the charge $(Z=22-42)$ or mass $(m=48-96)$ of the injected element, but this comparison does not rule out a $Z / m$ dependence since $Z / m \approx 1 / 2$ for all these elements.

Comparison of Ge injections into deuterium and helium discharges at 1.4 MA and $\overline{\mathrm{n}_{\mathrm{e}}}=1.2 \times 10^{19} \mathrm{~m}^{-3}$ (cases 1 and 2) shows no significant dependence of $\mathrm{D}$ or $\tau$ on the working gas species. In contrast, an increase in $\tau$ with the mass of the working gas was seen for impurities injected into hydrogen, deuterium, and helium plasmas in Alcator $C$ [26], but at substantially lower $Z_{\text {eff }}$ than in TFTR. A possible explanation of this discrepancy is that, unlike the case for Alcator $C$, collisions of injected impurities with carbon ions dominate collisions with working gas ions in these TFTR plasmas. 
Comparisons of shots at different plasma currents but similar $\overline{n_{e}}$, cases 3 and 4.2 and 8,9 and 10 , and 16 and 17 , show no significant dependence of $D$ on the current in the ranges $0.8-1.8 \mathrm{MA}$ at low $\overline{n_{\mathrm{e}}}$ and $2.2-2.5 \mathrm{MA}$ at high $\overline{n_{\mathrm{e}}}$. This is also seen in Fig. 7, a scatter plot of D versus current for the shors in Table III; $\overline{n_{e}}$ for each shot is indicated. Although the shots with the highest current are also those with the lowest $D$, these shots also have significantly higher $\overline{n_{e}}$ than those at lower current. An increase in $\tau$ with increasing current was seen in Alcator C [26] and in T-10 [27], but is not evident on TFTR.

The diffusion coefficient appears to decrease with $\overline{n_{e}}$ as the density limit is approached. Figure 8 is a plot of $D$ versus $\overline{n_{e}}$ for the shots in Table III, with the shots sorted by current. $D$ is smaller by approximately a factor of 2 in the discharges with $\overline{n_{e}} \geq 3 \times 10^{19} \mathrm{~m}^{-3}$ than in the lower $\overline{n_{e}}$ shots. However, the shots with $\overline{n_{e}} \geq 3 \times 10^{19} \mathrm{~m}^{-3}$ are all at densities which approach the observed density limits for TFTR plasmas with ohmic heating only. Thus, D appears to decrease as the density limit is approached at a given current. This is substantiated by comparison of cases 14 and 15 in Table III, $1.8 \mathrm{MA}$ deuterium plasmas at $\overline{n_{\mathrm{e}}}=2.3 \times$ $10^{19} \mathrm{~m}^{-3}$ and $3.7 \times 10^{19} \mathrm{~m}^{-3}$, and by comparison of cases $2,3,5$ and 6 in Table III, 1.4 MA helium plasmas at $\overline{n_{e}}=1.2-3.2 \times 10^{19} \mathrm{~m}^{-3}$. Increased confinement of injected impurities as the density limit is approached has been seen in TEXT [28], TEXTOR [29], and DITE [30]. As seen fron the lower density group of points in Fig. 8, there is no correlation between $D$ and $\overline{n_{e}}$ well below the density limir (i. e., $\overline{\mathrm{n}_{\mathrm{e}}} \leq 3 \times 10^{19} \mathrm{~m}^{-3}$ ).

The change in $Z_{\text {eff }}$ with density may play a role in the decrease of $D$ with $\overline{n_{e}}$. At constant current, $Z_{\text {eff }}$ decreases with increasing $\overline{n_{e}}$ in TFTR [31] with the result that the higher density points in Fig. 8 have the lowest values of $Z_{\text {eff. }}$ These shots also tend to have lower values of $D$. Figure 9 is a plot of $D$ versus $Z_{\text {eff }}$ with the value of $\overline{n_{e}}$ in each case indicated. There is no correlation between $D$ and $Z_{\text {eff }}$ for $Z_{\text {eff }} \geq 2.9$, but the shots with $Z_{\text {eff }} \leq 2.4$ have $D \leq 0.8 \mathrm{~m}^{2} / \mathrm{s}$. Thus, it is not clear that lower values of $D$ are correlated solely with higher values of $\overline{n_{e}} ; Z_{e f f}$ may also play. a role.

No correlations between $c_{\mathrm{V}}$ and any of the global plasma parameters mentioned above were found. In light of this and the uncertainties associated with the determination of $c_{y}$, it is possible only to conclude that a nonzero convective velocity is necessary to fit the data in most cases. 


\section{Summary}

The results of this study can be summarized as follows:

1. Modeling of time evolutions of lines emitted by injected impurities allows a spatially averaged diffusion coefficient and a convective peaking parameter to be measured.

2. Decay times of lines from edge ionization states are longer than predicted by transport models that fit the time evolutions of the central ionization states well. This is probably due to inaccuracies in the ionization and recombination rates.

3. Transport parameters were measured for $17 \mathrm{ohmically}$ heated TFTR discharges in both deuterium and helium with $\mathrm{I}_{\mathrm{p}}=0.8-2.5$ $\mathrm{MA}, \overline{\mathrm{n}_{\mathrm{e}}}=0.6-6.0 \times 10^{19} \mathrm{~m}^{-3}$, and $\mathrm{Z}_{\mathrm{eff}}=2-6$. Values of the diffusion coefficient, $D$, are in the range $0.5-1.5 \mathrm{~m}^{2} / \mathrm{s}$, considerably larger than the neoclassical values. Non zero inward convective velocities are found in most cases, and are similar to neoclassical values. In deuterium plasmas at $\mathrm{I}_{\mathrm{p}}=1.4 \mathrm{MA}$ and $\overline{\mathrm{n}_{\mathrm{e}}}=1.2 \times 10^{19} \mathrm{~m}^{-3}$, impurity and electron transport coefficients are of the same magnitude.

4. No significant differences in $D$ are found for injected elements in the range $Z=22-42$ and between deuterium and helium plasmas. $A$ strong scaling of $D$ with plasma current is not seen.

6. At a given current, $D$ is smaller by approximately a factor of two in discharges near the density limit than at lower densities. There is no correlation between $D$ and $\overline{n_{e}}$ for $\overline{n_{e}} \leq 3 \times 10^{19} \mathrm{~m}^{-3}$. However, the shots with low values of $Z_{\text {eff }}(\leq 2.4)$ have low values of $D$. Thus, it is not possible to attribute this decrease in D solely to an increase in $\overline{n_{e}}$ or a decrease in $Z_{e f f}$ on the basis of the present data.

7. No clear trends in the peaking parameter $c_{v}$ were found. 


\section{ACKNOWLEDGMENTS}

The authors would like to thank the entire TFTR group for their support of this work. Particular thanks are due to S. A. Cohen and R. J. Goldston for useful discussions and to $K . M$. Young.

This work was supported by the United States Department of Energy under contract No. DE-AC02-76-CHO-3073. 


\section{REFERENCES}

[1] Stratton, B. C., Cohen, S. A., Boody, F. P., Bush, C. E., Ellis III, R. et al., J. Nucl. Mater. 145-147 (1987) 587.

[2] Strachan, J. D., Chan, A., Nucl. Fusion 27 (1987) 1025.

[3] Mansfield, D. K., Efthimion, P. C., Hulse, R. A., Medley, S. S., Dylla. H. F., in Controlled Fusion and Plasma Physics (Proc. 14th Europ. Conf., Madrid. 1987) Vol. I1D, European Physical Society (I987) 314.

[4] Behringer, K. D., Carolan, P. G., Denne, B., Decker, G., Engelhardt. W. et al., Nucl. Fusion 26 (1986) 751.

[5] Abe, T., Aikawa, H., Akaoka, H., Akasaka, H., Akiba, M. et al., in Plasma Physics and Controlled Fusion Research 1986 (Proc. 1 lth Int. Conf., Kyoto, 1986) Vol. 1, IAEA, Vienna (1987) 217.

[6] Hulse, R. A., Nucl. Technol./Fusion 3 (1983) 259.

[7] Marmar, E. S., Cecchi, J. L., Cohen, S. A., Rev. Sci. Instrum. 46 (1975) 1149.

[8] Hawryluk, R. J., Arunasalam, V., Bell, M. G., Bitter, M., Blanchard, W. R. et al., in Plasma Physics and Controlled Nuclear Fusion Research 1986 (Proc. 1 th Int. Conf., Kyoto, 1986) Vol. 1, LAEA, Vienna (1987) 51.

[9] Fonck, R. J., Ramsey, A. T., Yelle, R. V., Appl. Opt. 21 (1982) 2115.

[10] Schwob, J. L.,Wouters, A. L., Suckewer, S., Finkenthal, M., Rev. Sci. Instrum. 58 (1987) 1601.

[11] Stratton, B. C., Fonck, R. J., Ida, K., Jaehnig, K. P., Ramsey, A. T., Rev. Sci. Instrum. 57 (1986) 2043.

[12] Post, D. E., Jensen, R. V., Tarter, C. B., Grasberger, W. H., Lokke, W. A., At. Data Nucl. Data Tables 20 (1977) 397.

[13] Hulse, R. A., Post, D. E., Mikkelsen, D. R., J. Phys. B, 13 (1980) 3895.

[14] Towner, H. H., Bell, M. G., Coonrod, J., Davis, S. L. et al., Bull. Am. Phys. Soc. 28 (1983) 1252.

[15] Mewe, R., Astron. Astrophys. 20 (1972) 215.

[16] Hulse, R. A., TFTR/JET INTOR Workshop on Plasma Transport in Tokamaks, July 1984, Rep. PPPL-2182, Princeton Plasma Physics Lab. (1985).

[17] TFR Group, Nucl. Fusion 23 (1983) 559.

[18] Lotz, W., Astrophys. J. Suppl. 14 (1967) 207. 
[19] Merts, A. L.. Cowan, R. D., Magee, N. H., The Calculated Power Output from a Thin Iron-Seeded Plasma. Rep. LA-6220-MS. Los Alamos Scientific L b. (1976).

[20] Janev, R. K., and Katsonis. K., Nucl. Fusion 27 (1987) 1493.

[21] Phaneuf, R., Oak Ridge National Lab., personal communication, 1987.

[22] Cohen. S. A., Princeton Plasma Physics Lab.. personal communication. 1989.

[23] Brice, D., Sandia National Lab., personal communication, 1988.

[24] Hasebe, Y., Kondoh, K., Morita, K., Sputtering of Metal Layers on Graphite at Elevated Temperatures, to be published in The Proceedings of the 1988 Particle-Surface-Interactions Meeting, Aachen, Germany.

[25] Rutherford, P. H. , Hirshman, S. P., Jensen, R., Post, D. E.. Seidl. F. G. P.. Impurity Transport in Tokamaks, Rep. PPPL-1297, Princeton Plasma Physics Lab. (1976).

[26] Marmar, E. S., Rice, J. E., Terry, J. L., Seguin, F. H., Nucl. Fusion 22 (1982) 1567.

[27] Bagdasarov, A. A., Vasin, N. L., Vershkov, V. A., Gegechikori, N. M., Zhuravlev, V. A. et al., in Plasma Physics and Controlled Nuclear Fusion Research 1984 (Proc. 10th Int. Conf., London, 1984) Vol 1, IAEA, Vienna (1985) 181.

[28] Isler, R. C., Rowan, W. L., Hodge, W. L., Phys. Rev. Lett. 55 (1985) 2413.

[29] Castracane, J., Demers, Y., Lönen, L., Pospieszczyk, A., Nucl. Fusion 27 (1987) 1921.

[30] Hawkes, N. C., Peacock, N. J., Bamsley, R., Fielding, S. J., Hugill, J., Johnson, P. C., in Controlled Fusion and Plasma Physics (Proc. 14th Europ. Conf., Madrid, 1987) Vol. 11D, European Physical Society (1987) 101.

[31] Stratton, B. C., Ramsey, A. T., Boody, F. P., Bush, C. E., Fonck. R. J. et al., Nucl. Fusion 27 (1987) 1147. 
Table I

Measured and Calculated Line Brighmesses in Example Discharge

\begin{tabular}{|c|c|c|c|}
\hline \multicolumn{4}{|c|}{ Time $=4.2 \mathrm{~s}, 0.1 \mathrm{~s}$ after injection } \\
\hline Line & Measured & Calculated & $\underline{\text { Ratio }}$ \\
\hline Ge XXI $196 \AA$ & 7.9 & 5.1 & 1.5 \\
\hline Ge XXII $226 \AA$ & 8.9 & 4.6 & 1.9 \\
\hline Ge $X X X 200 \AA$ & 3.7 & 2.4 & 1.5 \\
\hline Ge $X X X 123 \AA$ & 5.1 & 4.5 & 1.1 \\
\hline
\end{tabular}

Brightness units: $10^{12}$ photons $/ \mathrm{s}-\mathrm{cm}^{2}-\mathrm{sr}$ 
Table II

Effect of Ionization and Recombination Rate Variations on Line Brightnesses in Example Discharge

Ratio of Calculated Brightnesses to Reference Values $0.1 \mathrm{~s}$ After Injection

$\begin{array}{ccccc}\text { Line } & \text { Ion. } \times 2 & \text { Ion. } \times 0.5 & \text { Rec. } \times 2 & \text { Rec. } \times 0.5 \\ \text { Ge XXI 196 } & 0.3 & 2.7 & 2.7 & 0.2 \\ \text { Ge XXII 226 } & 0.2 & 1.2 & 1.1 & 0.2 \\ \text { Ge XXX 200 } & 1.0 & 0.4 & 0.6 & 1.1\end{array}$


Table III

Impurity Transport Parameters in Ohmically Heated TFTR Discharges

\begin{tabular}{|c|c|c|c|c|c|c|c|c|c|c|}
\hline 포 & TFCR shor & $\begin{array}{l}\text { Injected } \\
\text { element }\end{array}$ & $\begin{array}{l}\text { Working } \\
\text { gas }\end{array}$ & $\mathrm{I}, \mathrm{MA}$ & $\mathrm{B}_{\mathrm{t}}(\mathbb{\mathcal { T }})$ & $\overline{\mathrm{n}_{\mathrm{e}}}\left(10^{19} \mathrm{~m}^{-3}\right)$ & $z_{\text {eff }}$ & $I(s)$ & $\mathrm{D}\left(\mathrm{m}^{2} / \mathrm{s}\right)$ & $\mathbf{s}_{\mathbf{v}}$ \\
\hline i & 28716 & $\mathrm{Ge}$ & D & [.4 & 3.9 & 1.2 & 5.9 & 0.16 & 1.3 & 0.3 \\
\hline 2 & 31253 & $\mathrm{Ge}$ & $\mathrm{He}$ & t.4 & 4.0 & 1.2 & 5.5 & 0.16 & $0.9-1.1$ & $0-0.3$ \\
\hline 3 & 31260 & $\mathrm{Ge}$ & $\mathrm{He}$ & 1.4 & 4.0 & 2.1 & 3.5 & 0.15 & 1.5 & 0.5 \\
\hline 4 & 31266 & $\mathrm{Ge}$ & $\mathrm{He}$ & 1.0 & 4.0 & 2.1 & 2.9 & 0.14 & 1.5 & 0.5 \\
\hline 7 & 34873 & $\mathrm{Ge}$ & $\mathrm{He}$ & 1.4 & 3.9 & 2.6 & 3.4 & 0.16 & 1.3 & 0.4 \\
\hline & 31062 & $\mathrm{Cu}$ & $\mathrm{He}$ & 1.4 & 4.3 & 3.2 & 2.4 & 0.21 & 0.8 & 0.2 \\
\hline & 25234 & Ge & D & 0.8 & 4.7 & 0.6 & 4.1 & 0.17 & $1.3-1.5$ & $0.3-0.5$ \\
\hline 0 & 34695 & $\mathrm{Ge}$ & $\mathrm{He}$ & 0.8 & 3.9 & 1.1 & 3.2 & 0.14 & 1.3 & 0.4 \\
\hline & 31698 & $\mathrm{Cu}$ & D & 1.8 & 4.8 & 1.5 & 6.0 & 0.14 & $1.0-1.3$ & $0-0.3$ \\
\hline 10 & $31871^{*}$ & $\mathrm{Cu}$ & D & 1.2 & 4.9 & 1.3 & 4.6 & 0.16 & $0.8 \cdot 1.2$ & $0-0.5$ \\
\hline 1 & 31608 & $\mathrm{Ni}$ & D & 1.8 & 4.7 & 2.2 & 3.7 & 0.17 & $1.0-1.3$ & $0-0.3$ \\
\hline 12 & 28936 & $\mathrm{Ti}$ & He & 1.4 & 4.0 & 2.2 & $N A$ & 0.17 & 1.5 & 0.5 \\
\hline 13 & 28730 & Mo & $\mathrm{He}$ & 1.4 & 4.0 & 1.8 & 4.1 & 0.19 & $0.8-1.2$ & 0.0 .5 \\
\hline 4 & $14516^{* *}$ & Ge & D & 1.8 & 4.7 & 3.7 & 2.0 & 0.25 & $0.5-0.6$ & 0.0 .3 \\
\hline 15 & $14518^{* * *}$ & Ge & D & 1.8 & 4.7 & 2.3 & 3.7 & 0.21 & 1.5 & 0.6 \\
\hline & 24623 & Ge & $\mathrm{He}$ & 2.2 & 4.8 & 5.3 & 3.1 & 0.21 & 0.7 & 0.2 \\
\hline 1 & 24630 & $\mathrm{Ge}$ & He & 2.5 & 4.8 & 6.0 & 2.2 & 0.24 & $0.7-0.8$ & $0-0.2$ \\
\hline
\end{tabular}

- $\mathrm{R}=236 \mathrm{~cm}, \mathrm{a}=71 \mathrm{~cm}$

** movable limiter sho: $R=259 \mathrm{~cm}, a=80 \mathrm{~cm}$

NA: $Z_{\text {eff }}$ not available 


\section{FIGURE CAPTIONS}

Fig. 1. Plasma current $I_{p}$, line-averaged electron density $\overline{n_{e}}$. loop voliage. and total radiated power in the example discharge.

Fig. 2. Measured and calculated time evolutions of Ge XXII and Ge XXX lines in the example discharge. The calculations assumed a diffusion coefficient $\mathrm{D}=1.3 \mathrm{~m}^{2} / \mathrm{s}$ and and a convective peaking parameter $\mathrm{c}_{\mathrm{v}}=0.3$.

Fig. 3. Calculated distribution of ionization states in the example discharge $0.2 \mathrm{~s}$ after injection for $D=1.3 \mathrm{~m}^{2} / \mathrm{s}$ and $c_{y}=0.3$. (For clarity, states below $\mathrm{Ge}^{+20}$ are not shown.)

Fig. 4. Measured and calculated time evolutions of germanium lines in the example discharge. The ionization rates have been multiplied by 2.0 and 0.5 in the calculations, which assume $D=1.3 \mathrm{~m}^{2} / \mathrm{s}$ and $c_{\mathrm{V}}=0.3$.

Fig. 5. Measured and calculated time evolutions of germanium lines in the example discharge. The recombination rates have been multiplied by 2.0 and 0.5 in the calculations, which assume $D=1.3 \mathrm{~m}^{2} / \mathrm{s}$ and $\mathrm{c}_{\mathrm{v}}=0.3$.

Fig. 6. Measured time evolutions of germanium lines in the example discharge compared with neoclassical transport (solid line) and neoclassical transport plus an anomalous diffusion coefficient of $1.1 \mathrm{~m}^{2} / \mathrm{s}$ (dashed line).

Fig. 7. D versus current for ohmically heated TFTR discharges.

Fig. 8. D versus $\overline{n_{e}}$ for ohmically heated TFTR discharges.

Fig. 9. D versus $Z_{\text {eff }}$ for ohmically heated TFTR discharges. 
N
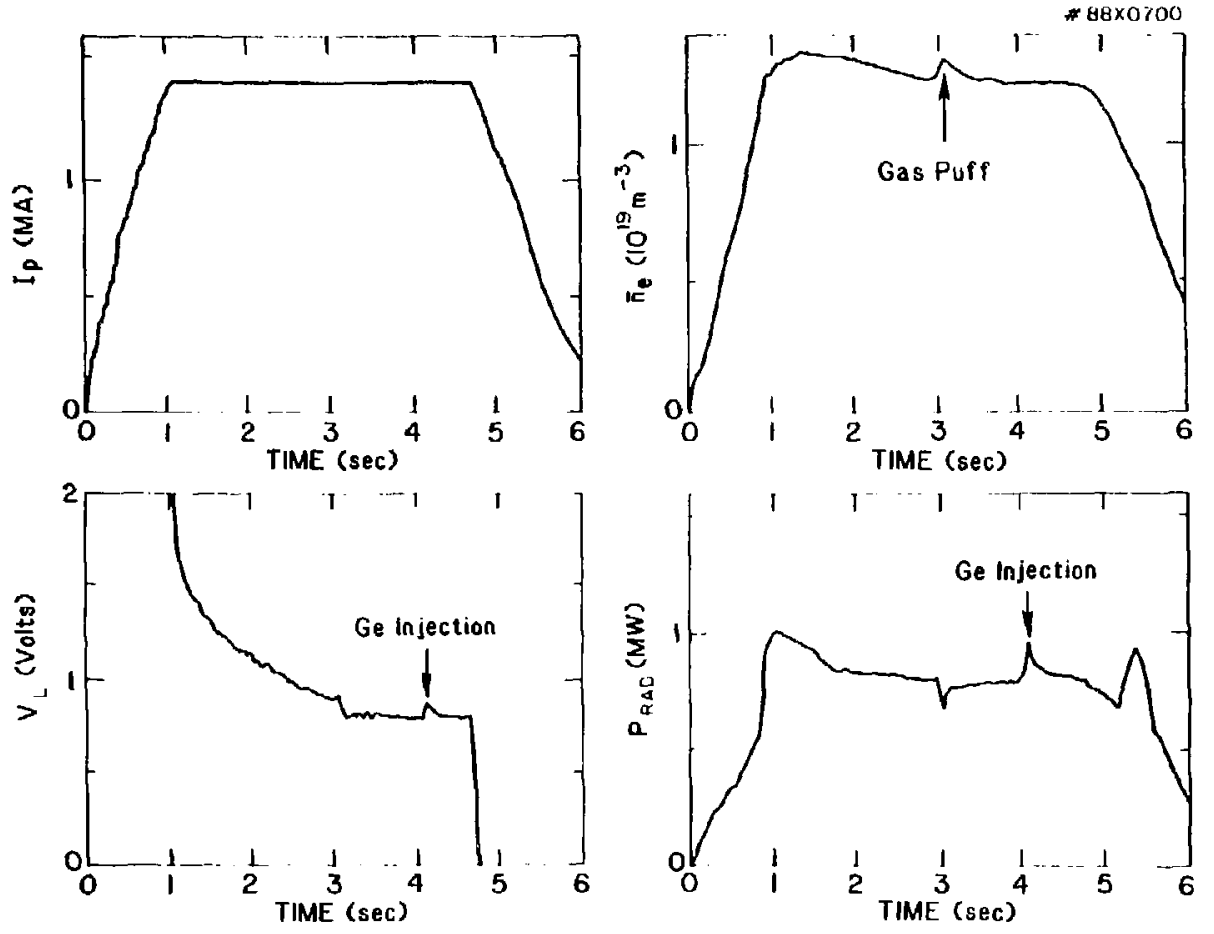


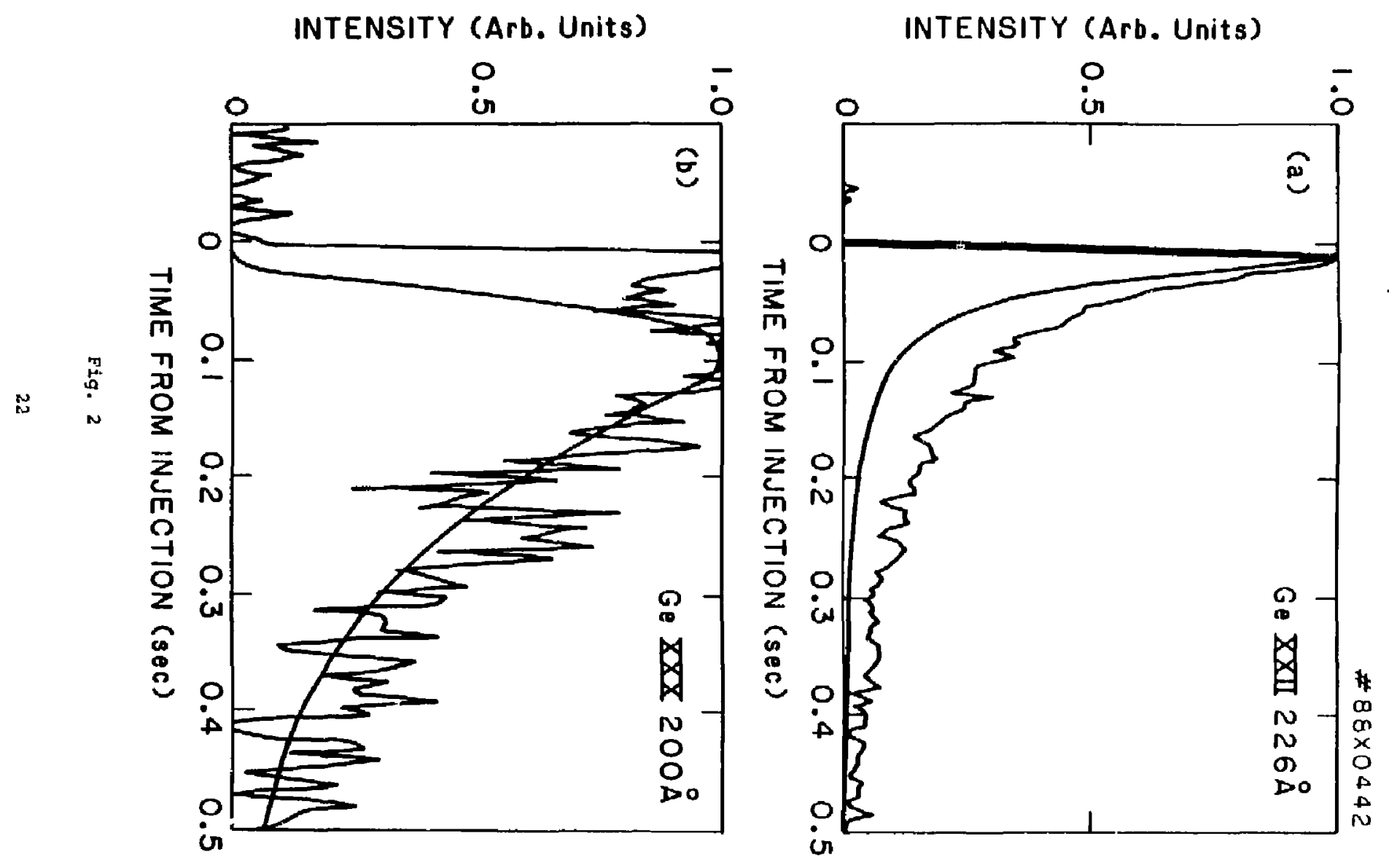




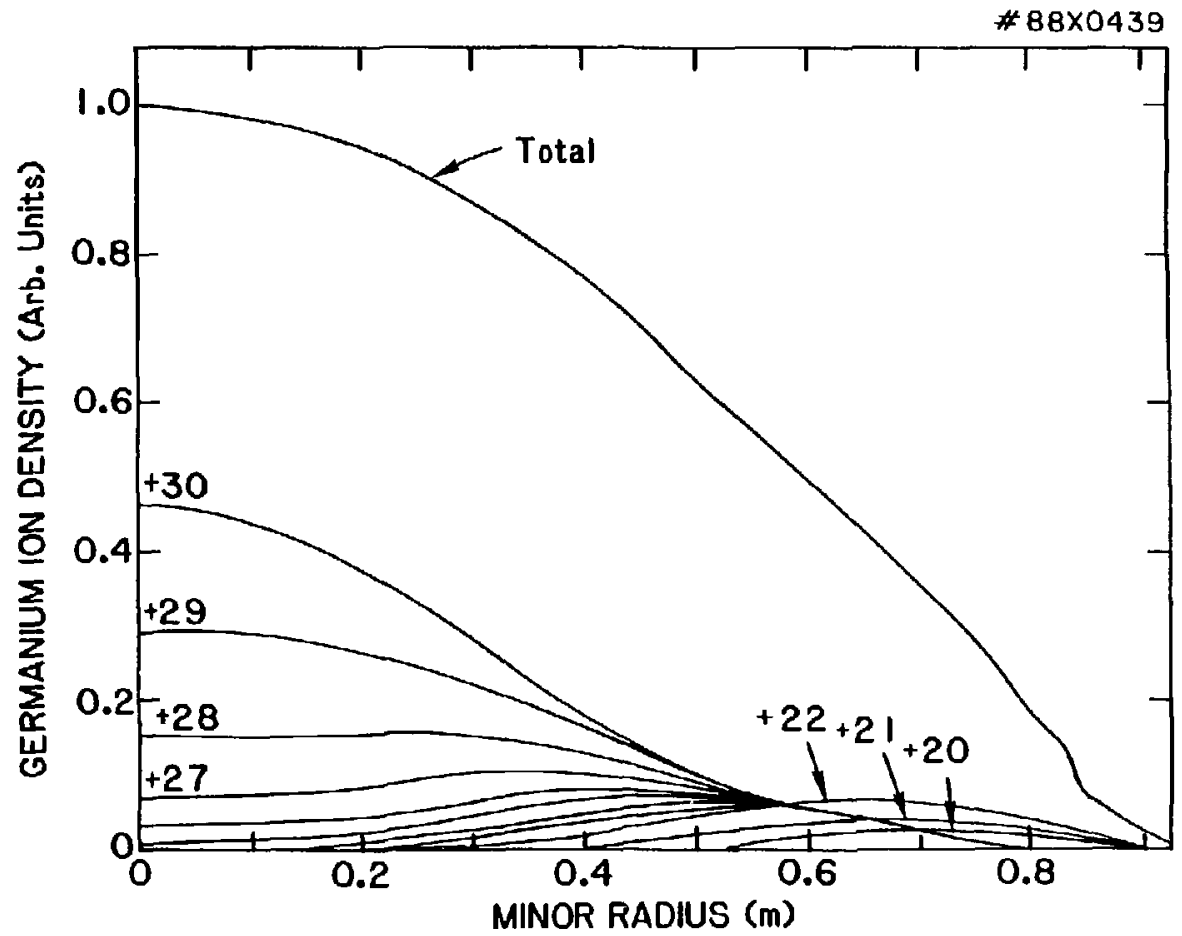

Fig. 3 

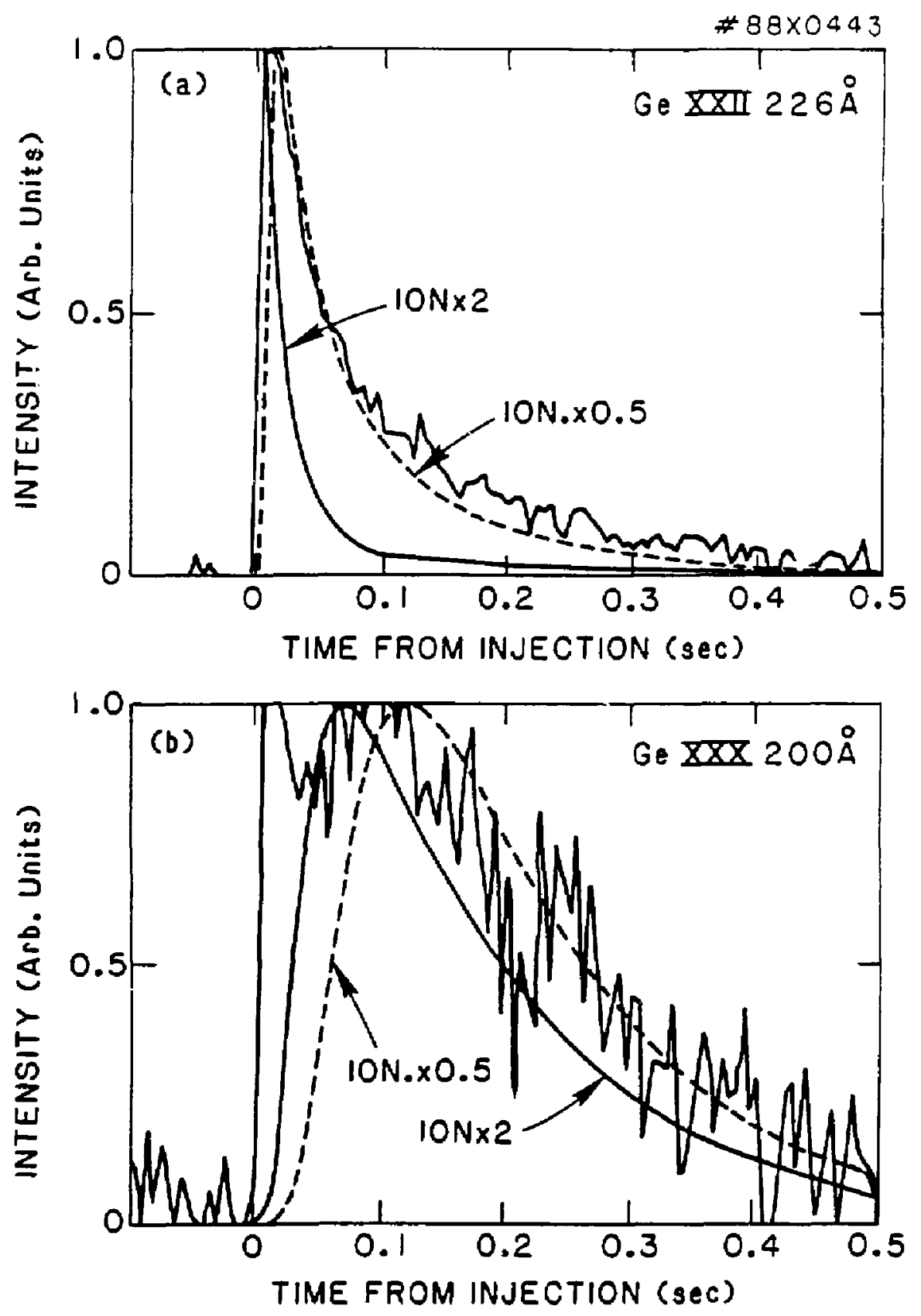

Fig. 4 

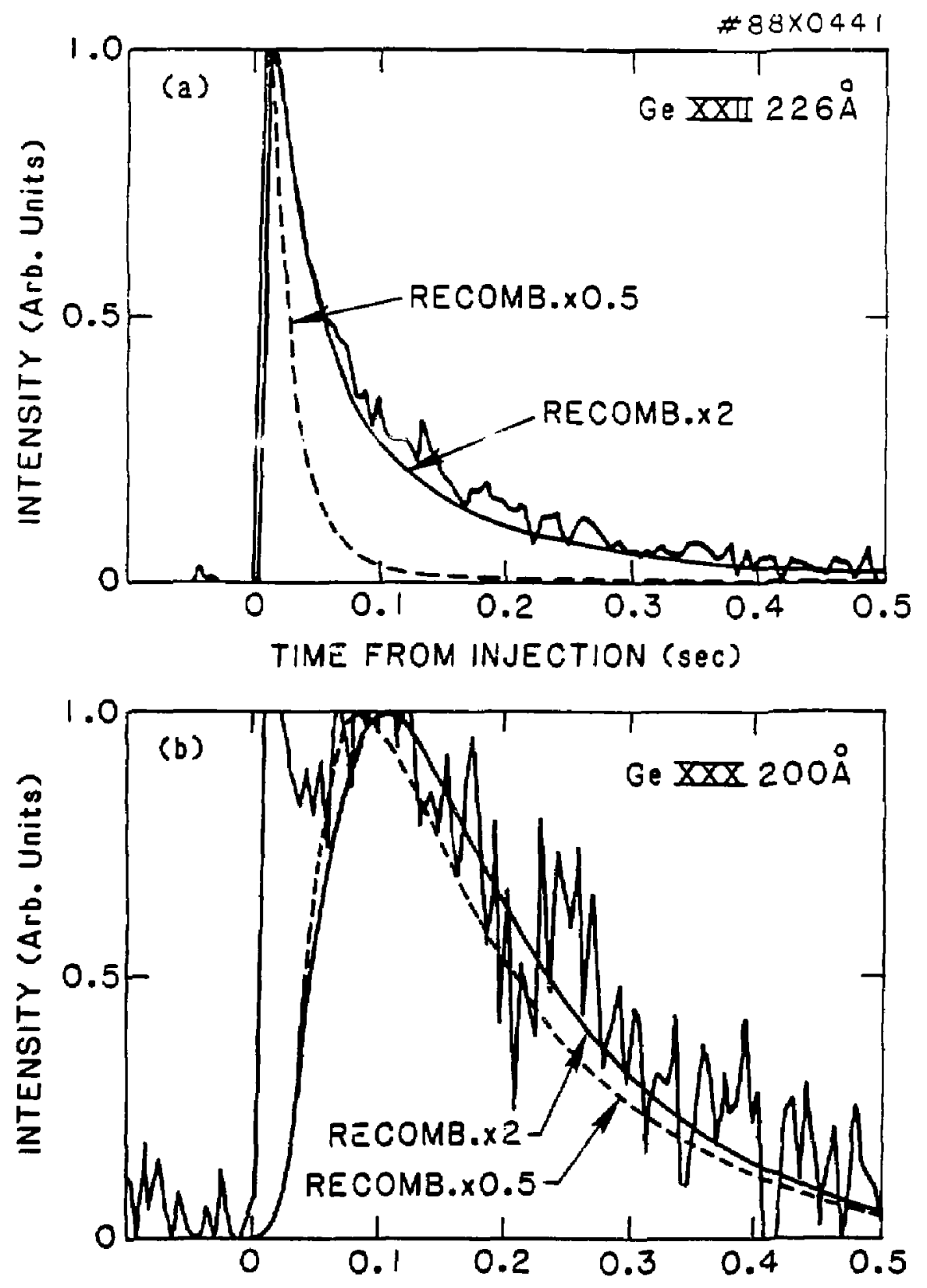

TIME FROM INJECTION (sec)

Fig. 5 

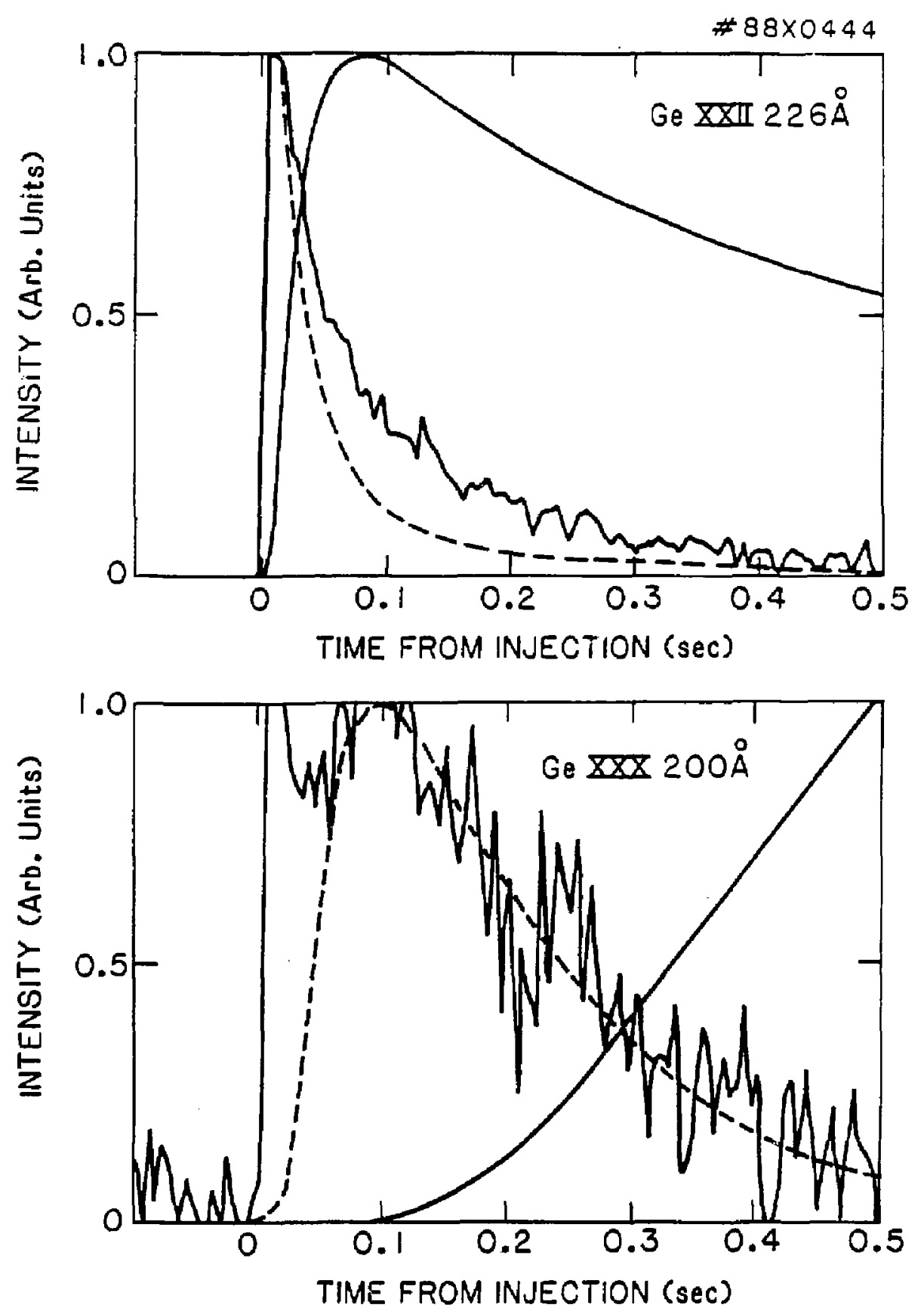

Eig. 6 


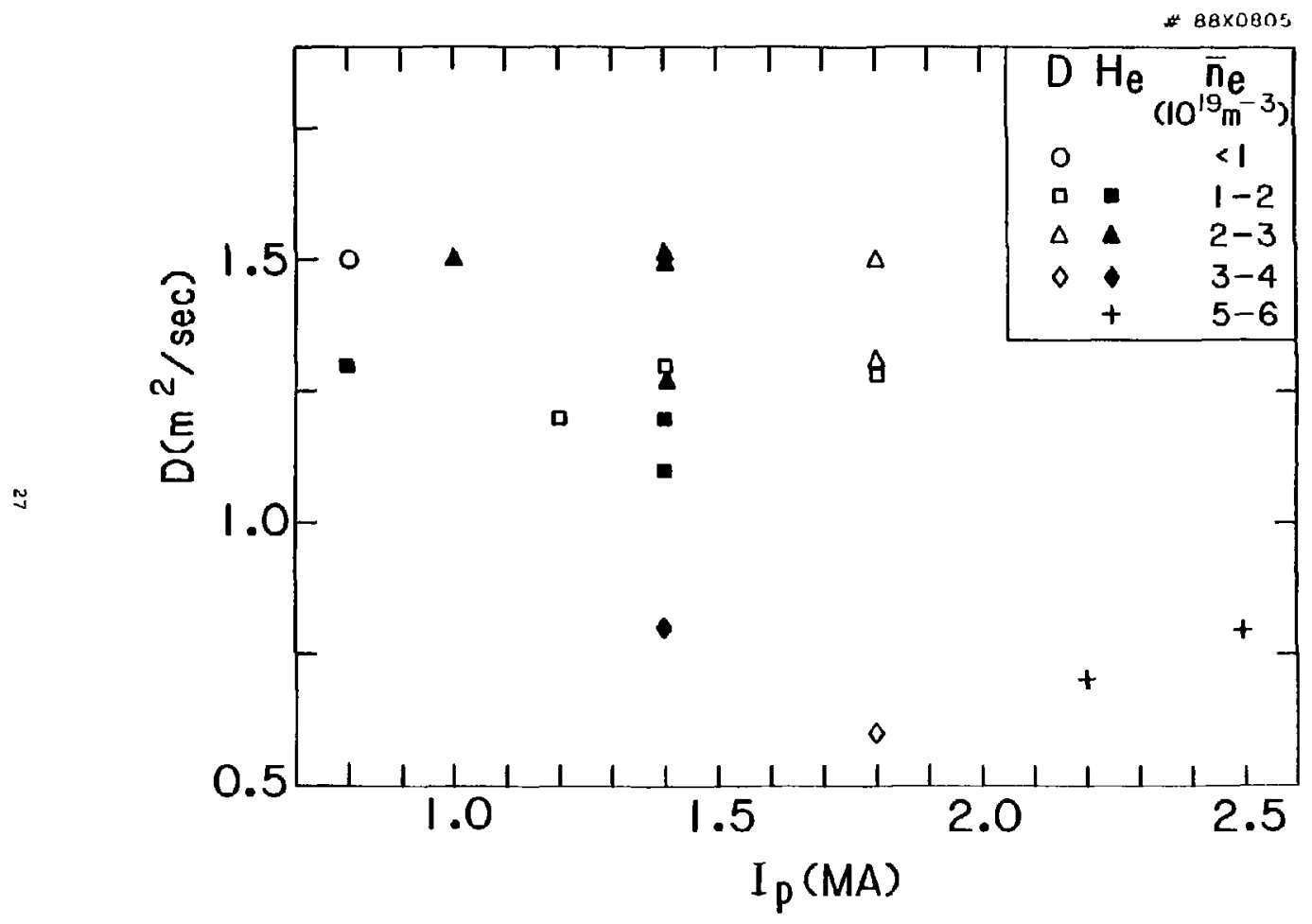

vig. ? 


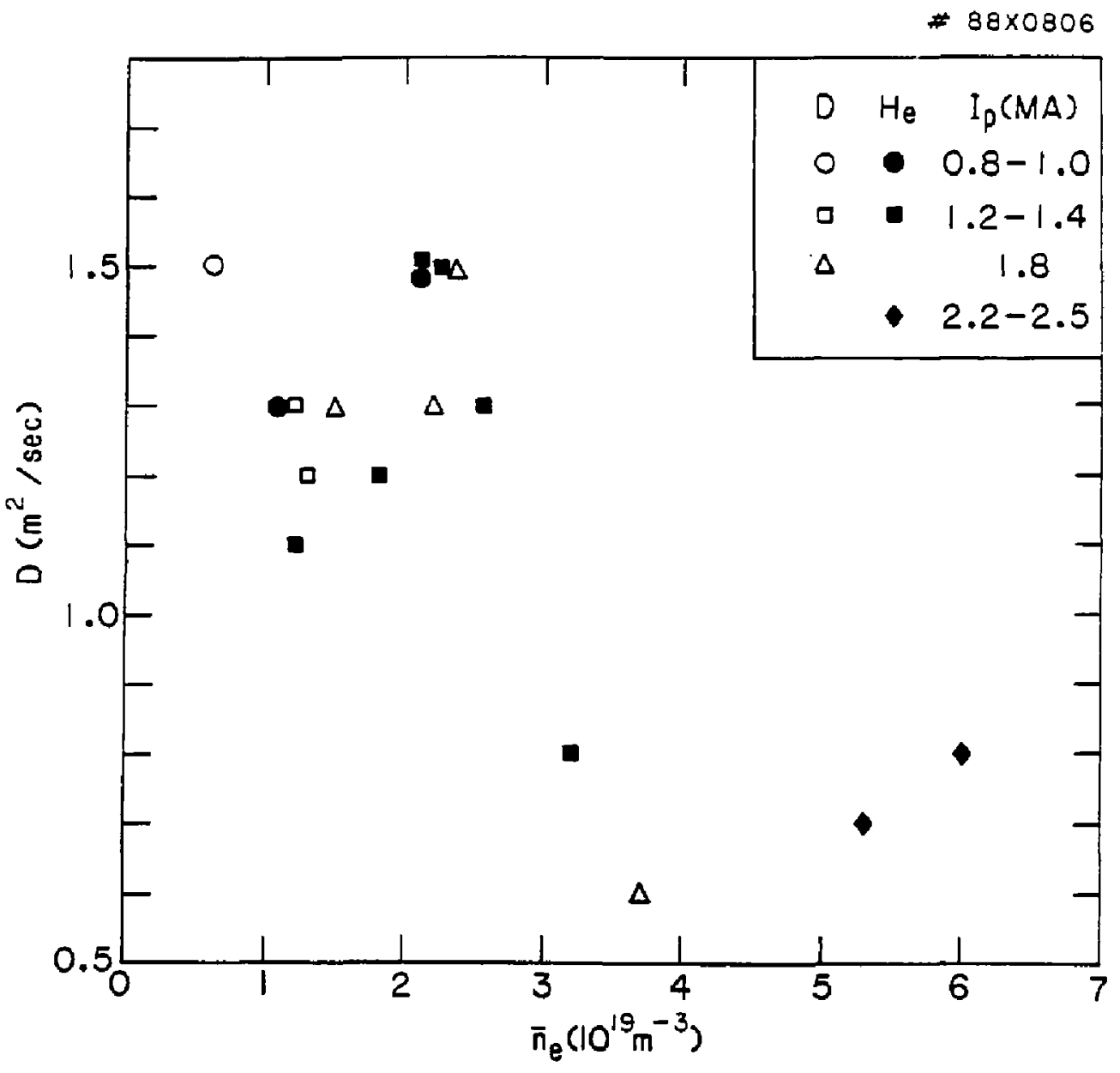

Fig. 8 


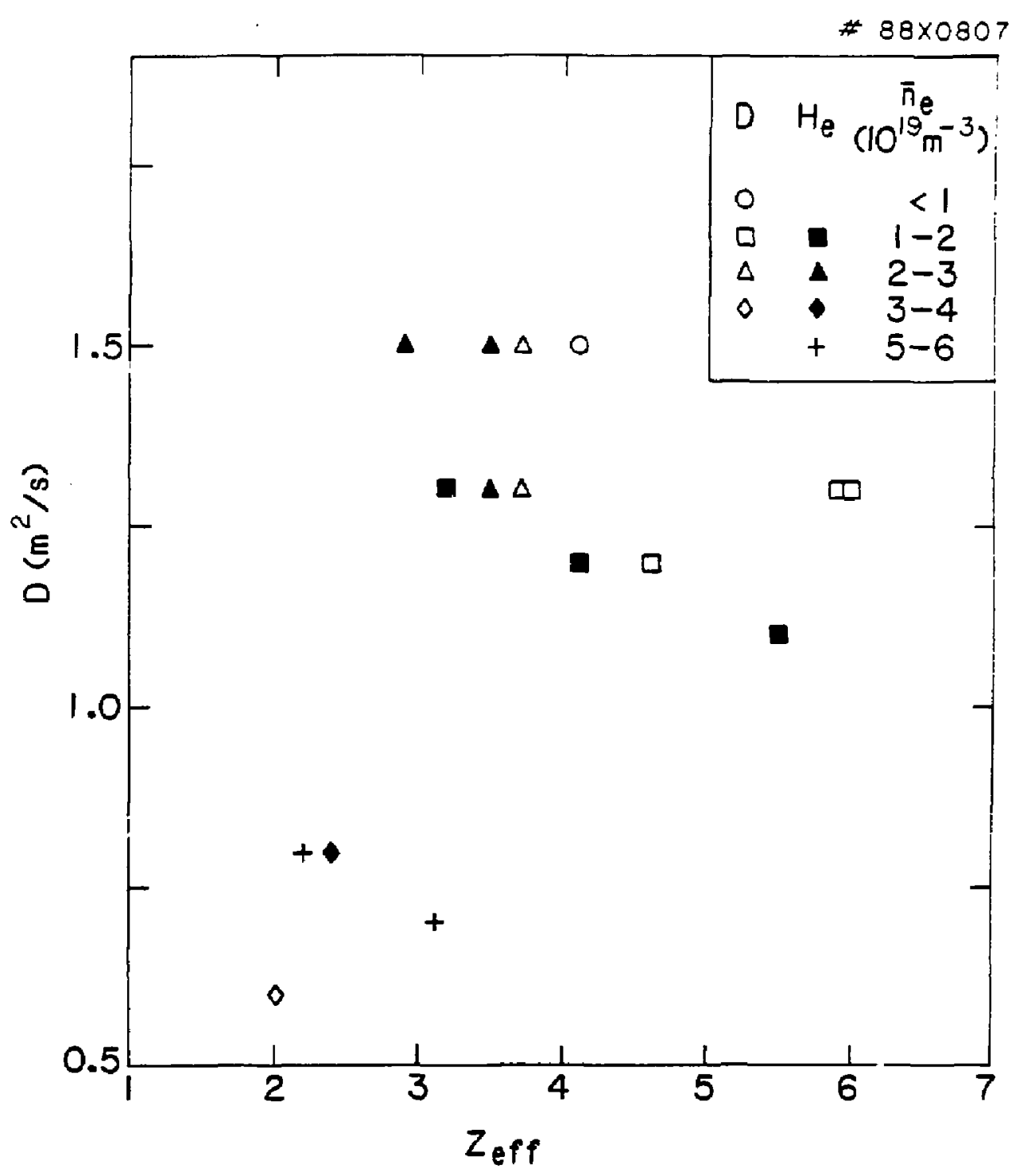

Fig. 9 